\title{
Towards a novel description of flavor dynamics in holographic QCD
}

\author{
Vasilis Niarchos* \\ Author affiliation \\ E-mail: niarchosephysics.uoc.gr
}

D-branes with a U-shaped geometry, like the D8 flavor branes in the Sakai-Sugimoto model of QCD, are encountered frequently in holographic backgrounds. The Dirac-Born-Infeld action, which is commonly used as an effective field theory description of open string theory at low energies, is not appropriate in this case, because it misses the dynamics of a (nonlocal) complex scalar mode. We review a recent attempt to incorporate this mode into an effective field theory description that extends the DBI action. Our results are relevant for the holographic description of chiral symmetry breaking and bare quark mass in QCD and open string tachyon condensation in curved backgrounds.

35th International Conference of High Energy Physics - ICHEP2010,

July 22-28, 2010

Paris France

${ }^{*}$ Speaker. 


\section{The problem}

The theoretical and phenomenological interest in analytic descriptions of the strong coupling dynamics of gauge theories, like QCD, is obvious. In recent years considerable effort has been devoted in the context of the AdS/CFT correspondence to the analysis of large- $N$ gauge theories whose strong coupling limit can be studied in the supergravity regime with the use of holographic methods. Large- $N$ QCD itself is not expected to admit a weakly curved gravitational dual; there are, however, theories with similar IR dynamics as QCD that have a dual weakly curved gravitational description. It is interesting to examine the lessons that we learn from such descriptions.

As a concrete example, let us consider the D4-D8- $\overline{\mathrm{D} 8}$ system in type IIA string theory (also known as the Sakai-Sugimoto holographic model of QCD [1]). The corresponding setup comprises of $N_{c}$ D4-branes, $N_{f}$ D8-branes and $N_{f}$ anti-D8-branes oriented in the following way

$$
\begin{aligned}
& N_{c} D 4: 01234 \\
& N_{f} D 8: 0012356789 \\
& N_{f} \overline{D 8}: 012356789
\end{aligned}
$$

The 4-direction is compactified with supersymmetry-breaking anti-periodic boundary conditions for the fermions. Hence, at low energies the open string theory on the D4-branes realizes the degrees of freedom of four-dimensional Yang-Mills theory. Chiral and anti-chiral quarks arise from D4-D8 and D4- $\overline{\mathrm{D} 8}$ open strings. We conclude that this setup realizes at low energies a theory with features very similar to those of QCD.

One can show that the IR dynamics of this model is captured at weak 't Hooft coupling by a non-local version of the Nambu-Jona-Lasinio model [2]. At strong 't Hooft coupling the IR dynamics is captured by QCD with $N_{f}$ flavors. In this regime, the $N_{c} \gg 1$ D4-branes dissolve into a closed string background captured by the supergravity solution of the Wick-rotated black D4brane. In the quenched approximation, where $N_{f} \ll N_{c}$, the $N_{f} \mathrm{D} 8-\overline{\mathrm{D} 8}$ pairs remain as D-branes embedded in this new background, but the non-trivial geometry forces them to reconnect and form $N_{f} \mathrm{U}$-shaped D8-branes. The open string theory on these branes captures holographically the flavor sector dynamics of the dual strongly coupled QCD-like gauge theory.

The formulation of a proper effective field theory description of the low-energy open string dynamics on these branes is a largely open problem. Typical problems whose solution depends on the ability to find this effective field theory description are: the computation of the order parameter of flavor chiral symmetry breaking, the incorporation of bare quark masses, the determination of the mesonic spectra etc.

Usually, in BPS D-brane configurations the degrees of freedom that dominate the low-energy physics are the low-lying massless modes of the open string: the transverse scalars and the gauge fields. The effective field theory of these modes is captured by the Dirac-Born-Infeld (DBI) action. This is not the case, however, for the above-mentioned U-shaped D8-branes for the following reasons. First, large accelerations develop in general near the turning point of a U-shaped brane and, by definition, the approximations that validate the use of the DBI action break down in that region. Second, when applied to the above holographic QCD setup the holographic dictionary implies that there has to be more than just transverse scalars and gauge fields in the low-energy description of the U-shaped D8-branes. A complex scalar field $\mathscr{T}$ must be present in the bi- 
fundamental representation of the flavor $U(1) \times U(1)$ global symmetry group. The normalizable branch of this field captures the vacuum expectation value (vev) of the dual meson-like operator that acts as the order parameter for chiral symmetry breaking. The non-normalizable branch captures the dual bare quark mass (see e.g. [3] for a related discussion).

A complex bi-fundamental scalar field $\mathscr{T}$ arises in the open string theory of the U-shaped D8brane as a low-lying mode in the NS - sector of an open string stretching between the asymptotic D8 and anti-D8-branches of the brane which lie at an arbitrary distance $L$. Even for large values of the separation $L$ this field can play an important role in the low-energy dynamics of the brane. With a non-trivial wavefunction in the holographic radial direction the effects of this field become important in the vicinity of the turning point where they are closely related with the geometric reconnection of the D8- $\overline{\mathrm{D} 8}$ pairs that capture the chiral symmetry breaking.

We conclude that the proper description of the reconnected D8-brane requires an extension of the DBI action that incorporates the bi-fundamental scalar mode $\mathscr{T}$. There are several technical complications in formulating such an action: $(i)$ our experience with effective actions of such scalar fields is in general limited in string theory (although significant progress has been achieved in the last decade, see below) and (ii) the scalar field, whose effects we want to capture, is a non-local field that arises from a long open string stretching along a macroscopic distance $L$.

We propose a new way to make progress in this problem. This is a brief review of the results obtained in [4].

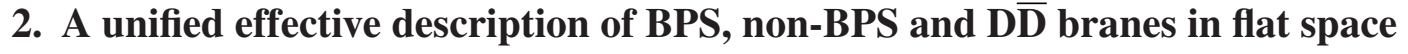

Our approach relies heavily on an effective field theory description of brane-antibrane dynamics put forward in [5]. The starting point of Ref. [5], which focused on D-branes in flat space, is the tachyon-DBI action (we set $\alpha^{\prime}=1$ )

$$
\begin{gathered}
S=-\int d^{p+1} \sigma V(T) \sqrt{-\operatorname{det}\left(\eta_{a b}+\partial_{a} X^{I} \partial_{b} X^{I}+F_{a b}+\partial_{a} T \partial_{b} T\right)}, \\
V(T)=\frac{\tau_{p}}{\cos \alpha T}, \alpha=1, \frac{1}{\sqrt{2}} \text { for bosonic, type II string theory }
\end{gathered}
$$

which describes the low-energy dynamics of a non-BPS D $p$-brane including the effects of the open string tachyon field $T$. This action has been derived in open string theory from first principles in $[6,7]$.

In this action, the real scalar field $T$, appears as an extra transverse scalar and the tachyon potential $V(T)$ as a $T$-dependent contribution to the dilaton. Taking this analogy a step further one can imagine an extended eleven-dimensional 'fictitious' spacetime with coordinates $X^{\mu}, T$ and metric and dilaton

$$
d s^{2}=g_{\mu \nu} d X^{\mu} d X^{v}+d T^{2}, e^{-\Phi(X, T)}=e^{-\Phi(X)} V(T)
$$

where BPS, non-BPS and brane-antibrane pairs arise as different solution curves of the DBI action formulated on the background (2.2).

Ref. [5] verified explicitly the validity of this picture in flat space. Non-BPS branes are automatically recovered as planar solutions oriented transverse to the extra coordinate $T$. BPS branes 

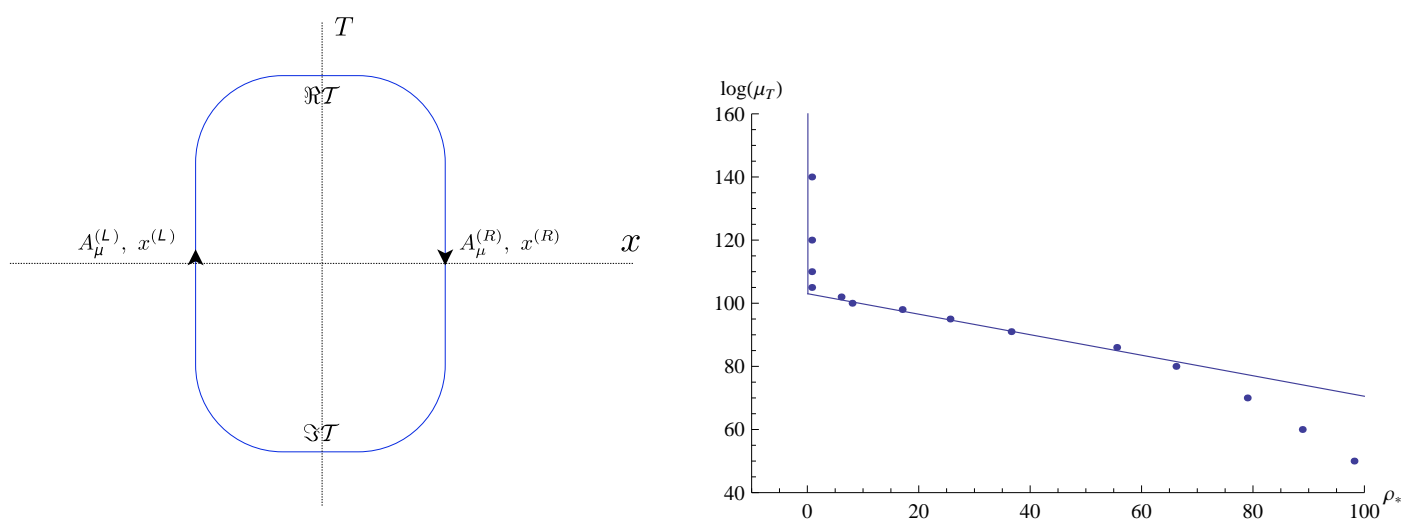

Figure 1: Left Figure: the tachyon-paperclip solution. Right Figure: a plot of $\log \mu_{T}$ as a function the radial turning point of the U-shaped brane in the background of NS5-branes. The solid curve is a fit based on the analytically expected result and the data points are based on a numerical evaluation of the equations of motion following from the action (3.2).

arise as planar solutions with one worldvolume direction parallel to $T$. To recover the DBI desciption of BPS branes it is crucial to notice that the normalizable worldvolume excitations are independent of the direction $T$ in the presence of the tachyon potential $V(T)$. Finally, one can recover the key properties of the brane-antibrane system at separation $L$ (namely, the right degrees of freedom and their interactions) as a closed 'tachyon-paperclip' curve in $(X, T)$ space (see the left plot in Fig. 1). In this description, different degrees of freedom arise from geometrically different parts of the solution, e.g. the left and right $U(1)$ gauge fields arise from the left and right $T$-oriented pieces of the paperclip. The non-local nature of the bi-fundamental complex scalar field $\mathscr{T}$, which arises as an emergent degree of freedom from the real $T$, is incorporated naturally. When $L<L_{\text {crit }}$, the solution is time-dependent and describes a condensing $\mathrm{D} \overline{\mathrm{D}}$ system $\left(L_{\text {crit }}=\pi \sqrt{2}\right.$ is the critical separation where $\mathscr{T}$ is massless).

\section{The NS5-D $p-\overline{\mathbf{D} p}$ system}

U-shaped branes, like the ones discussed above in the context of the Sakai-Sugimoto model, appear frequently in holographic backgrounds. The picture of section 2 suggests that the dynamics of U-shaped branes should be described by a 'radially condensing' tachyon-paperclip solution of the tachyon-DBI action. In order to test this idea, Ref. [4] applied the approach of section 2 to describe U-shaped (hairpin) D-branes in the background of NS5-branes.

This is a particularly interesting case that has many similar features to the Sakai-Sugimoto model. It is based on the following configuration of $k$ NS5-branes and $N_{f}$ D1 and anti-D1 semiinfinite branes $\left(6_{+}\right.$denotes a brane oriented along the positive $x^{6}$-direction)

$$
\begin{array}{cccc}
k N S 5 & : & 0 & 12345 \\
N_{f} D 1 & : & 0 & 6_{+} \\
N_{f} \overline{D 1}: & 0 & 6_{+}
\end{array}
$$

The 4-direction is again compactified with anti-periodic boundary conditions for the fermions. In the near-horizon background of the Wick rotated black NS5 branes, the D1-branes reconnect with 
the $\overline{\mathrm{D} 1}$-branes to form U-shaped D1-branes. A trivial T-duality along any of the directions (1235) relates this system to $\mathrm{D} p$-branes intersecting NS5-branes.

Since there are only NSNS-fluxes turned on in this case, it is possible to solve (both closed and open) string theory exactly in $\alpha^{\prime}$ with the use of the RNS formalism. In particular, we can see explicitly how a half-winding bi-fundamental scalar field $\mathscr{T}$ arises in the open string spectrum of the reconnected D1-branes and how it controls the open string theory on them. Our goal in Ref. [4] was to reproduce these results using the tachyon-DBI effective field theory.

The starting point is the tachyon-DBI action for a non-BPS D2-brane that wraps the background cigar-shaped geometry parametrized by $(\rho, \theta)$

$$
S=-\int d t d \rho d \theta \sinh \rho V(T) \sqrt{1+\frac{1}{k}\left(\partial_{\rho} T\right)^{2}+\frac{1}{k} \operatorname{coth}^{2} \rho\left(\partial_{\theta} T\right)^{2}} .
$$

The tachyon potential $V(T)$ is left as a free undetermined function.

Comparing with the exactly known solution in string theory one finds that

- The slope of the tachyon potential around $T=0$ is the same for all $k$ and coincides with the value of the flat space result.

- We can reproduce the asymptotic behavior of the bi-fundamental scalar field $\mathscr{T}$ at $\rho \gg \rho_{0}$ ( $\rho_{0}$ is the D1-brane turning point) with a tachyon-paperclip solution of the 'rolling tachyon' form $T \sim a+c(k) \log \cos \theta$, but this requires a modified $k$-dependent tachyon potential with large- $T$ behavior $V(T) \sim e^{-\frac{1}{\sqrt{k}} T}$.

- In the vicinity of the turning point we find a $\rho$-dependent elliptical solution

$$
\begin{aligned}
T= & A \sqrt{\rho-\rho_{0}} \cos \sigma+\ldots, \theta=B \sqrt{\rho-\rho_{0}} \sin \sigma+\ldots, \\
& 2 k B^{2} \tanh ^{2} \rho_{0}-A^{2} B^{2} \tanh \rho_{0}+2 A^{2}=0
\end{aligned}
$$

parametrized by two independent parameters.

- Similarly, there are two independent parameters controlling the leading and subleading branches of the solution $e^{T} \sim \mu_{T}+\ldots+M_{T} e^{-\rho}+\ldots$ at large $\rho$.

- Bootstrap methods in worldsheet CFT imply a specific relation between the boundary cosmological constant of the standard hairpin-brane and its turning point $\rho_{0}$ [8]. In terms of the leading branch coefficient $\mu_{T}$ of $e^{T}$ this relation translates to

$$
\log \mu_{T}\left(\rho_{0}\right)=x-z \log \sinh \left(y \rho_{0}\right), \quad y=k, z=\frac{1}{2(k-1)} .
$$

$x$ is a constant that depends on the normalization of $T$. The right plot in Fig. 1 depicts a set of data points determined numerically by solving the equations of motion of the action (3.2) for the randomly chosen value of $k=10$ and a fit based on the analytic result with parameters $y=6.5$ and $z=0.05$. The analytically expected parameters are $y=10$ and $z=$ $1 / 18 \sim 0.0556$. The apparent deviation in the vicinity of $\rho_{0} \sim 100$ is an expected feature of the approximations employed to obtain the numerical results. 


\section{Towards QCD}

The good qualitative and quantitative agreement of the tachyon-DBI-based results with the results expected from string theory suggest that this is a promising route towards the resolution of the problem outlined in section 1 .

A qualitatively similar picture is anticipated in the Sakai-Sugimoto model. This model has many similar features with the NS5-brane model discussed above, but also some interesting differences, e.g. the asymptotic separation $L$ of the U-shaped D8-branes is a modulus-dependent quantity in this case. A natural question is: does one need a more drastic modification of the tachyon-DBI potential $V(T)$ to reproduce this and other related features?

It would be very interesting to determine appropriate tachyon-paperclip solutions in the SakaiSugimoto model, to put constraints on the corresponding tachyon potential, and to determine the implications of this formalism for holographic QCD (namely fix the details of the holographic dictionary, compute the bare quark mass dependence, determine mesonic spectra etc.).

Beyond the very interesting applications to gauge theory we believe that this exercise will also be useful in uncovering new information about open string dynamics and tachyon condensation in curved backgrounds where an exact string theory treatment is currently out of reach.

\section{References}

[1] T. Sakai and S. Sugimoto, "Low energy hadron physics in holographic QCD," Prog. Theor. Phys. 113, 843 (2005) [arXiv:hep-th/0412141].

[2] E. Antonyan, J. A. Harvey, S. Jensen and D. Kutasov, "NJL and QCD from string theory," arXiv:hep-th/0604017.

[3] R. Casero, E. Kiritsis and A. Paredes, "Chiral symmetry breaking as open string tachyon condensation,” Nucl. Phys. B 787, 98 (2007) [arXiv:hep-th/0702155].

[4] V. Niarchos, "Hairpin-Branes and Tachyon-Paperclips in Holographic Backgrounds," Nucl. Phys. B 841, 268 (2010) [arXiv:1005.1650 [hep-th]].

[5] D. Erkal, D. Kutasov and O. Lunin, "Brane-Antibrane Dynamics From the Tachyon DBI Action," arXiv:0901.4368 [hep-th].

[6] D. Kutasov and V. Niarchos, "Tachyon effective actions in open string theory," Nucl. Phys. B 666, 56 (2003) [arXiv:hep-th/0304045].

[7] V. Niarchos, "Notes on tachyon effective actions and Veneziano amplitudes," Phys. Rev. D 69, 106009 (2004) [arXiv:hep-th/0401066].

[8] K. Hosomichi, “N=2 Liouville Theory with Boundary,” JHEP 0612, 061 (2006)

[arXiv:hep-th/0408172]. 